\title{
Multi Wavelet for Image Retrival Based On Using Texture and Color Querys
}

\author{
K. Joseph Bhushanam ${ }^{1}$, P. Darwin ${ }^{2}$ \\ ${ }^{I}$ M.tech.Student, Godavari institute of ENG \& Technology, Rajahmundry \\ ${ }^{2}$ Associate Professor, Godavari institute of ENG \& Technology, Rajahmundry
}

\begin{abstract}
Efficient, texture representation is important, in search and retrieval of similar texture patterns from a large image database. There has been research on texture feature extraction by finding the Spatial/frequency distribution of the patterns with tools like the Gabor filters, Pyramid-structured wavelet transform, and Multi wavelet transform. In this paper, content based image retrieval method is used, to retrieve the images from the database according to their similarity to the Query image. The aim of this project is to retrieve the images using multi wavelet. Firstly, we calculate the multi wavelet of all images in database.secondly, to calculate the energy and then distance between query and database images.
\end{abstract}

Keywords: Retrieving Images, CBIR (Content Based Image Retrieval) system, distance and energy

\section{Introduction}

Content-Based Image Retrieval (CBIR) is the process of retrieving images from a database on the basis of features that are extracted automatically from the images themselves [2]. A CBIR method typically converts an image into a feature vector representation and matches with the images in the database to find out the most similar images. In the last few years, several research groups have been investigating content based image retrieval. A popular approach is querying by example and computing relevance based on visual similarity using low-level image features like color histograms, textures and shapes. Text-based image retrieval can be traced back to the 1970 's; images were represented by textual descriptions and subsequently retrieved using a text-based database management system [3]. Content-based image retrieval utilizes representations of features that are automatically extracted from the images themselves. Most of the current CBIR systems allow for querying-by-example, a technique wherein an image (or part of an image) is selected by the user as the query. The system extracts the features of the query image, searches through the database for images with similar features, and displays relevant images to the user in order of similarity to the query [6][7][8][9].

Image Retrieval aims to provide an effective and efficient tool for managing large image databases. With the ever growing volume of digital image generated, stored, accessed and analyzed.

The paper is organized as follows. Gabor wavelet is presented in Section II. Section III describes the Multi wavelet. The texture feature calculation is presented in Section IV. Distance calculation are made in Section V. Results are shown in section VI. Section VII describes the conclusion.

\section{Gabor Wavelet}

The Fourier transform has been the most commonly used tool for analyzing frequency properties of a given signal, while after transformation, the information about time is lost and it's hard to tell where a certain frequency occurs. To solve this problem, we can use kinds of time-frequency analysis techniques. The Gabor transform, named after Dennis Gabor, is a special case of the transform. It is used to determine the sinusoidal frequency and phase content of local sections of a signal as it changes over time. The function to be transformed is first multiplied by a Gaussian function, which can be regarded as a window function, and the resulting function is then transformed with a Fourier transform to derive the time-frequency analysis. The window function means that the signal near the time being analyzed will have higher weight. The Gabor transform of a signal $\mathrm{x}(\mathrm{t})$ is defined by this formula:

$$
G_{x}(t, f)=\int_{-\infty}^{\infty} e^{-\pi(\tau-t)^{2}} e^{-j 2 \pi f \tau} x(\tau) d \tau
$$

The Gaussian function has infinite range and it is impractical for implementation. However, a level of significance can be chosen (for instance 0.00001) for the distribution of the Gaussian function.

$$
\begin{cases}e^{-\pi a^{2}} \geq 0.00001 ; & |a| \leq 1.9143 \\ e^{-\pi a^{2}}<0.00001 ; & |a|>1.9143\end{cases}
$$


Outside these limits of integration $|a|>1.9143$, the Gaussian function is small enough to be ignored. Thus the Gabor transform can be satisfactorily approximated as

$$
G_{z}(t, f)=\int_{-1.9143}^{1.9143} e^{-\pi(\tau-t)^{2}} e^{-j 2 \pi f \tau} x(\tau) d \tau
$$

\section{Drawbacks}

1) In this technique takes more execution time

2) The accuracy of similarity image retrieval is low.

To solve this problem using multi wavelet technique.

\section{Multi Wavelet Transform}

Multiwavelets were defined using several wavelets with several scaling functions [7]. Multiwavelets have several advantages in comparison with scalar wavelet [8]. A scalar wavelet cannot possess all these properties at the same time. On the other hand, a multiwavelet system can simultaneously provide perfect representation while preserving length (Orthogonality), good performance at the boundaries (via linearphase symmetry), and a high order of approximation (vanishing moments) [9]. Thus multiwavelets offer the possibility of superior performance and high degree of freedom for image processing applications, compared with scalar wavelets.

During a single level of decomposition using a scalar wavelet transform, the 2- D image data is replaced by four blocks corresponding to the sub bands representing either low pass or high pass in both dimensions. These sub bands are illustrated inFig. 3 .

The multi-wavelets used here have two channels, so there will be two sets of scaling coefficients and two sets of wavelet coefficients. Since multiple iteration over the low pass data is desired, the scaling coefficients for the two channels are stored together.

Likewise, the wavelet coefficients for the two channels are also stored together. The flow chart of proposed method as shown in fig 1 .

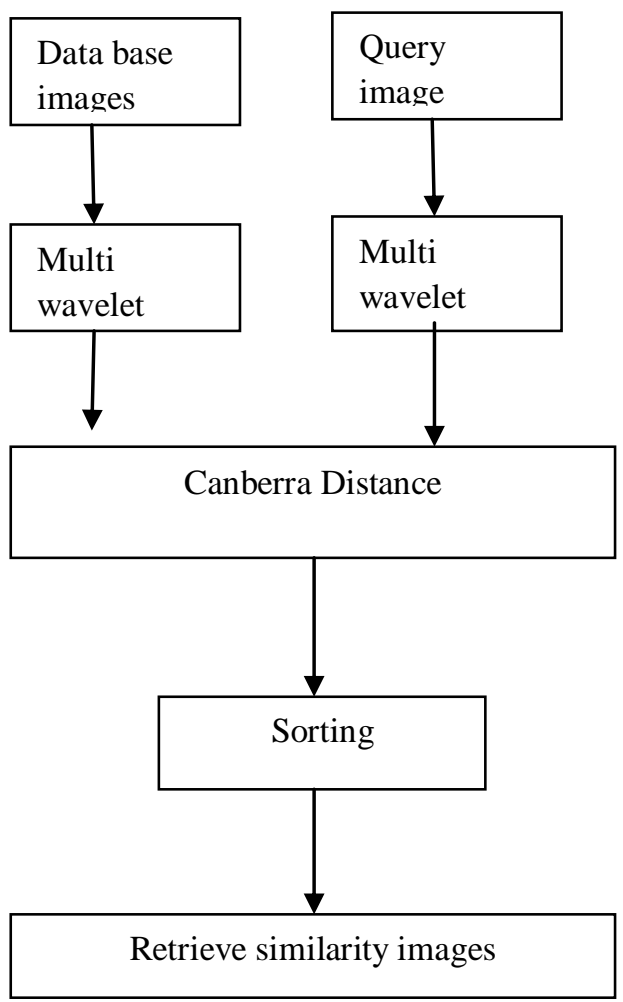

Fig.1.Flowchart of proposed algorithm

The procedure of proposed algorithm as shown below

1) Input as Query image I as taken

2) Convert RGB to HSV

3) Apply pyramid structural wavelet to hue color 
4) Calculate the features(color, texture and edge) values of the image

5) Similarity comparisons between input image and database by using Euclidian distance.

6) Sorting the distance values

7) Finally relevant images are retrieved with respect to corresponding query image I.

8) Repeat step 1 to 7 for another query image.

\section{Texture Feauture Calculation}

For the texture characteristic four descriptors are used that are: contrast, entropy, energy and inverse differential moment. We have used the Euclidian distance to measure the similarity between the Query image and database image. First apply the Multi wavelet to get the image into eight sub-images.

Calculate the energy of all decomposed images at the same scale, using:

$$
E=\frac{1}{M N} \sum_{i=1}^{m} \sum_{j=1}^{n}|X(i, j)|
$$

where $\mathrm{M}$ and $\mathrm{N}$ are the dimensions of the image, and $\mathrm{X}$ is the intensity of the pixel located at row $\mathrm{i}$ and column $\mathrm{j}$ in the image map. Using the above algorithm, the energy levels of the sub-bands is calculated, and further decomposition of the low-low sub-band image is also done This is repeated three times, to reach third level decomposition. These energy level values are stored to be used in the Euclidean distance algorithm.

\section{Distance Calculation}

In this section, we take Canberra distance between two vectors $\mathrm{p}$ and $\mathrm{q}$ is shown below

$$
d^{C A D}(\mathbf{p}, \mathbf{q})=\sum_{i=1}^{n} \frac{\left|p_{i}-q_{i}\right|}{\left|p_{i}\right|+\left|q_{i}\right|},
$$

\section{Results}

In the experiment various images are used in the MATLAB program. The results of two different test images using Gabor wavelet and multi wavelet as shown below.
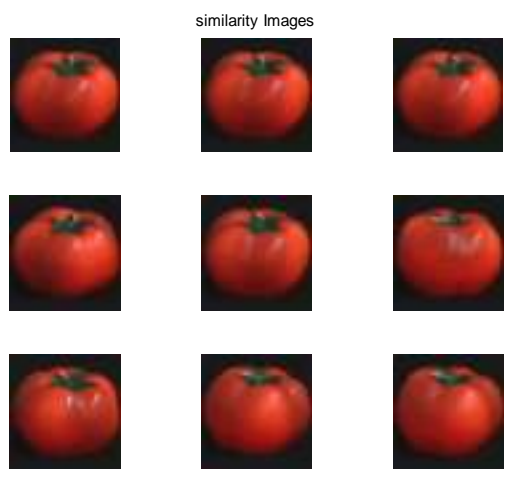

Fig.2.rotating coil tomoto using multiwavelet

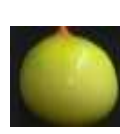

$$
\text { similarity Images }
$$
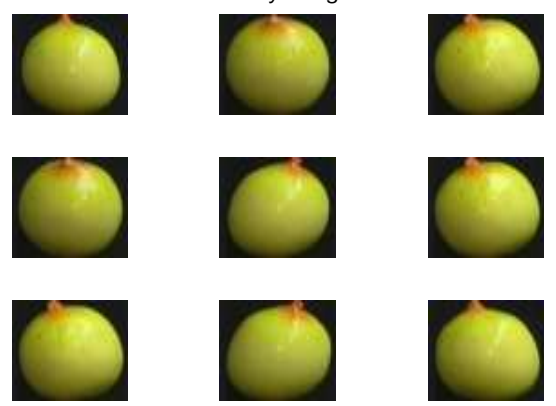

Fig.3.rotating coil mango using multiwavelet 

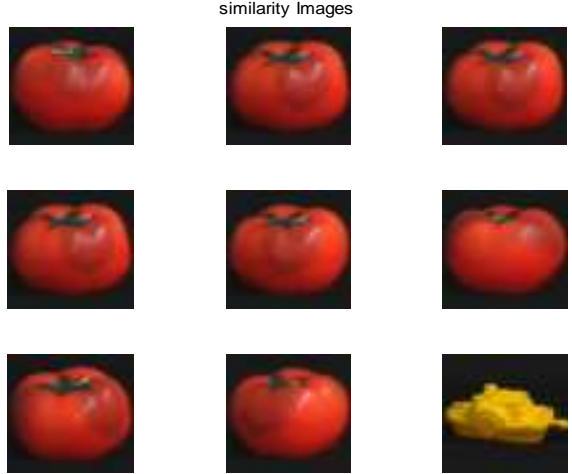

Fig.4.rotating coil tomoto using Gabor wavelet
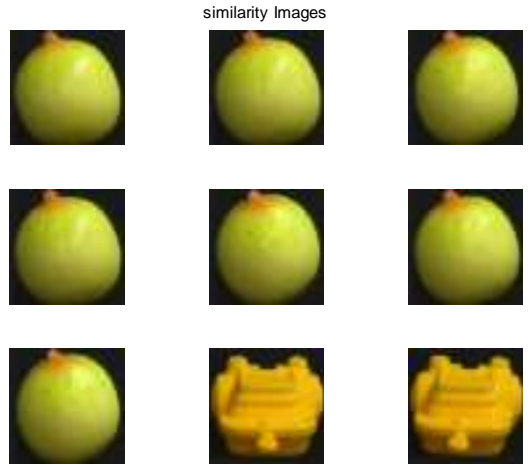

Fig.5.rotating coil mango using gabor wavelet

\begin{tabular}{|c|c|c|c|}
\hline technique & $\begin{array}{c}\text { Total } \\
\text { database } \\
\text { images }\end{array}$ & accuracy & time \\
\hline $\begin{array}{c}\text { Gabor } \\
\text { wavelet }\end{array}$ & 530 & $91.5 \%$ & $120 \mathrm{sec}$ \\
\hline $\begin{array}{c}\text { Multi } \\
\text { wavelet }\end{array}$ & 530 & 98.5 & $12 \mathrm{sec}$ \\
\hline
\end{tabular}

Table 1. Shows the comparison between Gabor wavelet and Multi wavelet

\section{Conclusion}

This paper attempts to evaluate the performance of the CBIR system on sample datasets of images using Multi wavelet. Multi wavelet transform is the development of wavelet transform and construct wavelet with two scaling function. Multi wavelet transform has many relatively good properties such as symmetry, short support, orthogonality and high order vanishing moments. The system gives good results on the tests conducted. Further tests must be conducted on various and large databases to have a more accurate evaluation. The indexation technique is a crucial part in a CBIR system.

To have a more powerful and efficient retrieval system for image and multimedia databases, content based queries must be combined with text and keyword predicates.

\section{References}

[1] J Eakins, M E Graham, Content-based Image Retrieval, A report to the JISC Technology Applications Programme, 1999.

[2] Thomas S. Huang, Yong Rui, and Shinh-Fu Chang, Image retrieval: Past, Present and Future, International Symposium on Multimedia Information Processing, 1997.

[3] M. Myron Flickner, H. Sawhney, W. Niblack, J. Ashley, Q. Huang, B. Dom, M. Gorkani, J. Hafner, D. Lee, D.Petkovic, D.Steele and P.Yanker, "Query by image content: The QBIC system", In IEEE Computer, pp. 23-31, Sept.1995

[4] Patrick M. Kelly, Michael Cannon and Donald R. Hush, Query by Image Example: The CANDID approach. In Storage and Retrieval for Image and Video Databases III, volume 2420, pp 238-248, SPIE, 1995

[5] Chad Carson, Serge Belongie, Hayit Greenspan, Jitendra Malik, Blobworld: Image segmentation using Expectation-Maximization and its application to image querying, Third International Conference on Visual Information Systems, 1999

[6] M. Das, E. M. Riseman, and B. A. Draper. Focus: Searching for multi-colored objects in a diverse image database, In IEEE Conference on Computer Vision and Pattern Recognition, pp 756-761, Jun 1997 